\title{
Establishment of standardised SLA/LP immunoassays: specificity for autoimmune hepatitis, worldwide occurrence, and clinical characteristics
}

\author{
M Baeres, J Herkel, A J Czaja, I Wies, S Kanzler, E L R Cancado, G Porta, M Nishioka, \\ T Simon, C Daehnrich, W Schlumberger, P R Galle, A W Lohse
}

See end of article for authors' affiliations

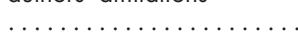

Correspondence to: Dr A W Lohse,

I Department of Medicine Johannes

Gutenberg-University

Langenbeckstr 1, D-55101

Mainz, Germany;

lohse@mail.uni-mainz.de

Accepted for publication 23 October 2001

\begin{abstract}
Background: Antibodies to soluble liver antigen/liver pancreas (SLA/LP) are specific markers of autoimmune hepatitis. Their target antigen has recently been cloned.

Aims: To establish standardised immunoassays using the recombinant antigen, and to assess the frequency and significance of seropositivity in patients from different countries.

Methods: An enzyme linked immunoassay was developed using purified recombinant antigen and validated by testing sera from 200 healthy blood donors and 1026 patients with various liver and nonliver diseases. The assay was then applied to 454 sera from 419 patients with autoimmune hepatitis from different countries. All sera were also tested by inhibition immunoassay and western blot. Results: Antibodies were reliably detected by the recombinant immunoassay and occurred exclusively in patients with autoimmune liver disease. Twenty three of 149 patients from the USA (15\%), 23/132 from Brazil (17\%), $21 / 108$ from Germany (19\%), and 2/30 from Japan (7\%) were seropositive. Clinical features at presentation were similar between seropositive and seronegative patients. However, relapse after corticosteroid withdrawal or during maintenance therapy occurred more commonly in seropositive patients.

Conclusions: Antibodies to SLA/LP can be reliably detected by these standardised immunoassays based on recombinant antigen. Antibodies to SLA/LP occur with similar frequencies in different geographical regions, races, and age groups, and are of exquisite diagnostic specificity. Whether SLA/LP positive patients represent a clinically distinct subgroup remains to be determined; relapse during treatment reduction appeared to be more common in the SLA/LP group.
\end{abstract}

$\mathrm{T}$ he diagnosis of autoimmune hepatitis (AIH) is based on a combination of clinical, laboratory, and histological findings. ${ }^{12}$ The presence of autoantibodies is an important component of the diagnosis but the most commonly detected autoantibodies, antinuclear antibodies (ANA) and smooth muscle antibodies (SMA), are not specific for the disease. ${ }^{3}$ Antibodies to liver-kidney microsome type 1 (anti-LKMl) occur in a small subgroup of patients with AIH ${ }^{4}$ but they are also found in chronic hepatitis $\mathrm{C}^{5-10}$ and in severe rejection after liver transplantation. ${ }^{11}$ In contrast, antibodies to soluble liver antigen (SLA) and liver-pancreas antigen (LP) have been described as specific for autoimmune liver disease. ${ }^{12}{ }^{13}$ Several reports have shown that many patients with AIH negative for ANA and SMA show positive SLA reactivity or LP reactivity, making these antibodies an important diagnostic marker. ${ }^{12-15}$ Recently, antibodies to SLA and LP (anti-SLA/LP) have been shown to be identical, and their specificity for AIH and overlap syndromes of primary biliary cirrhosis (PBC) and AIH has been confirmed. ${ }^{16}$

ANA, SMA, and anti-LKMl are detected by assays based on indirect immunofluorescence. ${ }^{17}$ Antibodies to SLA/LP do not react in this fashion and thus escape normal serological screening. Other standardised immunoassays have not been available due to uncertainties concerning the target antigen. A few laboratories have used an inhibition enzyme linked immunosorbent assay (iELISA), as originally described by Manns and colleagues, ${ }^{12}$ which tests binding inhibition of an indicator SLA positive serum. This assay however crucially depends on the quality and labelling of the indicator serum. Consequently, it is not suitable for generalised or standardised testing. The recent identification and cloning of the target molecule, a presumed $50 \mathrm{kDa}$ cytosolic enzyme of as yet unclear function, has made it possible to develop standardised test systems for the detection of anti-SLA/LP. ${ }^{16}$ The identity of this cloned molecule as the target antigen has recently been confirmed $^{18}$; SLA/LP seems to be identical to a UGA suppressor tRNA associated protein that has been described as an autoantigen in AIH. ${ }^{19} 20$

In this report, we describe the development and validation of a standardised and simple ELISA for the detection of SLA/LP antibodies which utilises recombinant SLA/LP protein (rELISA), as well as a confirmatory western blot. Furthermore, we estimated the relative frequency of SLA/LP positive $\mathrm{AIH}$ in four different geographical regions and ethnic populations. Finally, we compared the clinical characteristics and response to therapy of SLA/LP positive and SLA/LP negative AIH patients.

\section{METHODS}

Serum samples

To develop and evaluate the rELISA for SLA/LP autoantibodies based on the recombinant antigen, sera from 95 patients with autoimmune hepatitis and 200 healthy blood donors were tested. Findings were compared with results from the iELISA

Abbreviations: SLA/LP, soluble liver antigen/liver pancreas; $\mathrm{AlH}$, autoimmune hepatitis; ANA, antinuclear antibodies; SMA, smooth muscle antibodies; LKM, liver/kidney microsome; PBC, primary biliary cirrhosis; iELISA, inhibition based enzyme linked immunosorbent assay; rELISA, recombinant antigen based enzyme linked immunosorbent assay; RU, relative units; SDS-PAGE, sodium dodecyl sulphate-polyacrylamide gel electrophoresis; PBS, phosphate buffered saline; lg, immunoglobulin. 

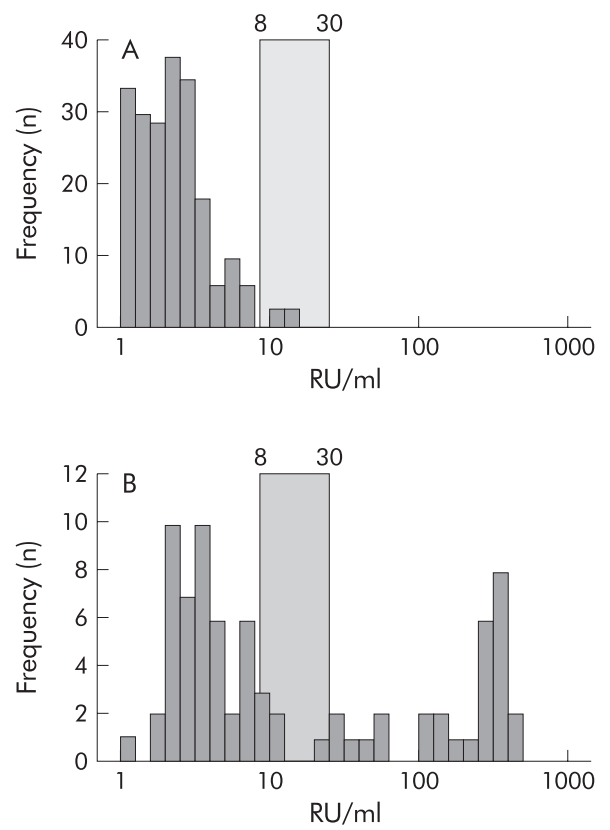

Figure 1 Characteristics of the standardised immunoassay with recombinant soluble liver antigen/liver pancreas (SLA/LP) antigen. The sera of 200 healthy blood donors (A) and 95 patients with autoimmune hepatitis (B) were screened for autoantibodies to SLA/LP. A borderline range was defined as between 8 and 30 relative units $(\mathrm{RU}) / \mathrm{ml}$ to exclude false positives.

and western blot using the recombinant antigen. Specificity was confirmed by testing a total of 1026 serum samples from consecutive patients with suspected or various known liver diseases seen at the outpatient clinic of the 1st Department of Medicine, Johannes Gutenberg-University, Mainz, Germany, including 235 consecutive general medical patients seen as inpatients.

To estimate the frequencies of anti-SLA/LP in patients with AIH from different countries, 454 sera from 419 patients treated at four different referral centres were tested by rELISA and western blotting. A total of 149 specimens were obtained from 149 patients diagnosed at the Mayo Clinic, Rochester, Minnesota, USA. One hundred and fifty four specimens were obtained from 132 patients diagnosed at the Liver Unit, Sao Paulo University, Brazil. Forty three specimens were obtained from 30 patients diagnosed at the Kagawa Medical University, Kagawa, Japan, and 108 specimens were obtained from 108 patients diagnosed at the Johannes Gutenberg-University, Mainz, Germany.

All patients satisfied the diagnostic criteria for AIH, according to the International Autoimmune Hepatitis Group. ${ }^{2}$ Sera were sent frozen to the laboratory at Johannes Gutenberg University, Mainz, Germany, and assessments were performed in batches.

\section{Recombinant SLA/LP antigen}

Recombinant SLA/LP antigen was expressed in Escherichia coli with the described J-Dl cDNA clone ${ }^{16}$ which encodes the shorter of two splice variants of the SLA/LP target antigen. J-Dl cDNA was subcloned into vector pET24d (Novagen, Bad Soden, Germany) providing a carboxy terminal histidine tag. Thus it was guaranteed that only complete proteins containing the carboxy terminal epitope, which is dominant in immunorecognition by SLA/LP antibodies, were purified.

SLA/LP protein was then expressed in E coli as an insoluble protein present in inclusion bodies. After lysis of bacteria, inclusion bodies were purified by centrifugation and washing. Following solubilisation of the inclusion bodies in $6 \mathrm{M}$ guanidine hydrochloride, recombinant SLA/LP protein was purified

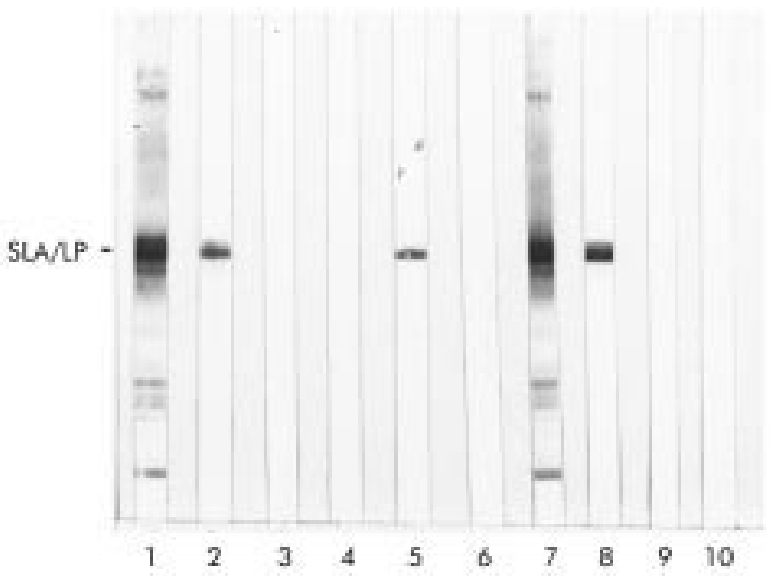

Figure 2 Confirmation of the ELISA assay by immunoblotting of recombinant soluble liver antigen/liver pancreas (SLA/LP) antigen. Reactivity of patient sera $(1: 200)$ to recombinant SLA/LP antigen was tested by western blotting. Results of immunoblotting were comparable with those in the ELISA assay with recombinant SLA/LP antigen. Shown are the reactivities in western blotting of representative sera that were either positive in the ELISA assay (lanes 1 and 2), negative (lanes 3 and 4), or borderline positive (lanes 5 and 6). The corresponding reactivity in the ELISA assay of the serum shown in lane 1 was above 200 relative units (RU)/ml; that of serum in lane 2 was $95 \mathrm{RU} / \mathrm{ml}$; serum in lane 3 was below $2 \mathrm{RU} / \mathrm{ml}$; serum in lane 4 was $2 \mathrm{RU} / \mathrm{ml}$; serum in lane 5 was $22 \mathrm{RU} / \mathrm{ml}$ (remaining positive in western blotting); and serum in lane 6 was $16.25 \mathrm{RU} / \mathrm{ml}$ (negative by western blotting). As controls we used: a positive standard serum (lane 7; above $200 \mathrm{RU} / \mathrm{mll}$ ); a monoclonal antibody to the recombinant SLA/LP protein from mouse (lane 8; diluted 1:1500); and the secondary antibodies alone (anti-human in lane 9; anti-mouse in lane 10).

by metal chelate affinity chromatography and subsequent cation exchange chromatography. Lastly, the product was dialysed against $20 \mathrm{mM}$ Tris $\mathrm{HCl}$ pH 7.6/0.01 mM EDTA/0.02\% sodium dodecyl sulphate (SDS). Analysis by SDSpolyacrylamide gel electrophoresis (SDS-PAGE) showed more than $95 \%$ purity.

\section{ELISA based on recombinant SLA/LP (rELISA)}

Recombinant SLA/LP antigen was used to coat maxisorp ELISA plates (Nunc, Roskilde, Denmark) at an antigen concentration of $0.02 \mu \mathrm{g}$ per well. After being post-coated with $0.1 \%$ casein/phosphate buffered saline (PBS), plates were incubated with $100 \mu \mathrm{l}$ of each patient's serum (diluted 1:100 in buffer) for 30 minutes at room temperature and then washed three times. Afterwards, plates were incubated with $100 \mu \mathrm{l}$ of peroxidase conjugated, rabbit derived antibody to human immunoglobulin G (IgG) for another 30 minutes, washed again, and then treated with $100 \mu \mathrm{l}$ of chromogene/substrate solution (tetramethylbenzidine/hydrogen peroxide). This treatment induces a change from colourless to blue in anti-SLA/LP positive wells. Fifteen minutes later, the reaction was stopped by $100 \mu \mathrm{l}$ of stop solution ( $1 \mathrm{~N}$ phosphoric acid), and plates were read at $\lambda 450 \mathrm{~nm}$ (and a reference wavelength of $\lambda>620 \mathrm{~nm}$ ) in a Spectra Mini (Tecan, Crailsheim, Germany).

As there was no international World Health Organization standard serum for detection of anti-SLA/LP, the ELISA was standardised using a serum sample from one patient with high titre anti-SLA/LP. This serum sample was defined as reference serum for reasons of availability, reactivity, and absence of other major autoantibodies. Calibration was done in relative units per millilitre $(\mathrm{RU} / \mathrm{ml})$ using three dilutions $(2 \mathrm{RU} / \mathrm{ml}, 20$ $\mathrm{RU} / \mathrm{ml}$, and $200 \mathrm{RU} / \mathrm{ml}$ ), resulting in a linear range of reactivity. The sera of 200 healthy blood donors (fig 1A) and 95 sera from patients with AIH (fig 1B), 29 of whom had anti-SLA/LP as defined by iELISA, were used to calibrate the rELISA assay. 


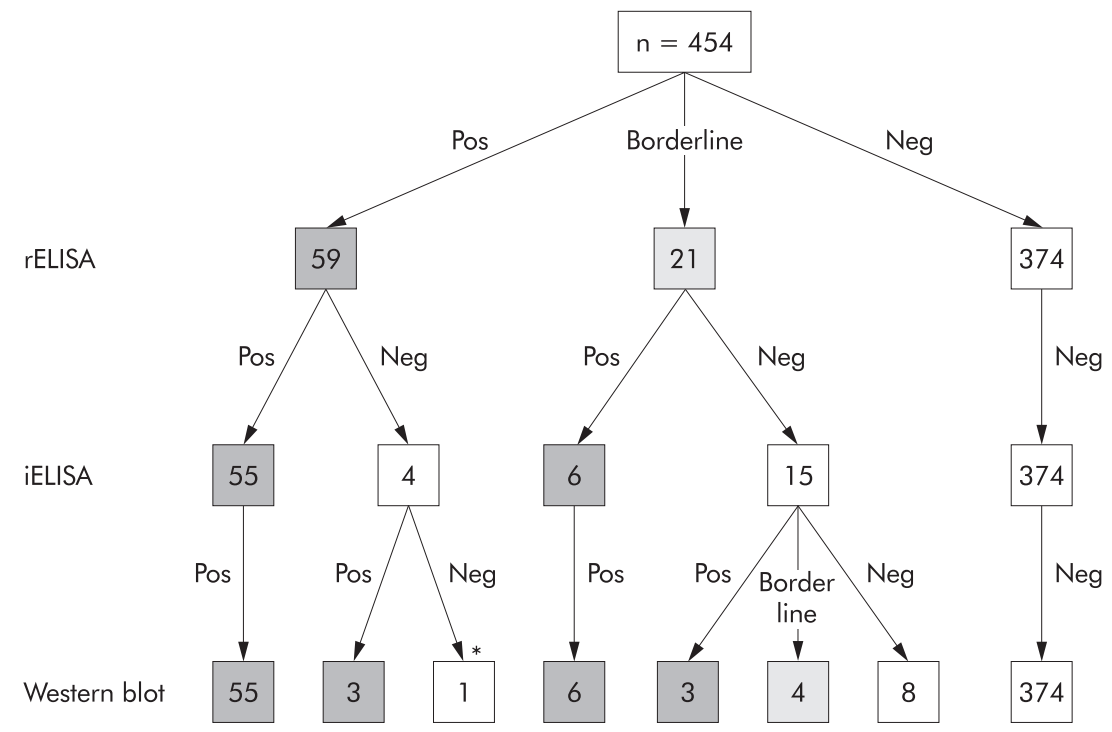

Figure 3 Incidence of soluble liver antigen/liver pancreas (SLA/LP) autoantibodies in 454 serum samples from four different countries (USA, Brazil, Germany, and Japan). Sera were tested by the recombinant antigen based ELISA (rELISA) and inhibition based ELISA (iELISA) and results were confirmed by western blotting. Shown is the incidence of seropositive (pos), borderline positive, and seronegative (neg) findings. * Serum sample that gave falsely positive results in various other ELISA assays also.

Subsequently, the assay was validated by testing 1026 non-selected sera, mostly from patients with various liver diseases, including 235 sera from general medical patients. By comparison with the iELISA and western blotting, the lowest SLA/LP autoantibody positive test serum gave $8.6 \mathrm{RU} / \mathrm{ml}$ in the rELISA, and the highest seronegative test serum showed 27.7 $\mathrm{RU} / \mathrm{ml}$. Thus specimens were classified as positive $(>30$ $\mathrm{RU} / \mathrm{ml})$, negative $(<8 \mathrm{RU} / \mathrm{ml})$, and borderline $(8-30 \mathrm{RU} / \mathrm{ml})$ reactive for anti-SLA/LP by the recombinant antigen based ELISA. Nine of the 1026 test sera were positive in the rELISA. Eight of the positive specimens were from patients with known SLA/LP positive AIH, and one was from a patient with the overlap syndrome of PBC and secondary AIH. ${ }^{16}{ }^{21}$ All nine sera were confirmed positive by western blot and all nine patients fulfilled the diagnostic criteria of AIH. ${ }^{2}$ Six further sera were borderline positive by the rELISA. One of these was clearly positive by western blot, and this patient was also independently diagnosed as suffering from AIH. The other five sera were negative by western blotting.

ELISA based on inhibition of indicator serum (iELISA) To allow comparison with earlier studies on SLA autoantibodies, all samples from AIH patients were also tested by an ELISA based on the inhibition of reactivity of a labelled indicator serum, as described previously. ${ }^{12}$ The ability of the patient's serum to inhibit the binding of the indicator serum to rat liver homogenate was utilised as a measure of anti-SLA/LP reactivity. The performance and validity of this assay system have been described previously. ${ }^{12}{ }^{14}$ As reliability of the assay varies and depends on the quality of the indicator serum, patient sera were only considered positive if inhibition was above two SDs of normal control sera (at least $40 \%$ inhibition) on two separate occasions. In this fashion, specimens could be classified as positive or negative for anti-SLA/LP by the inhibition based ELISA.

\section{Western blotting}

All sera testing positive or borderline positive in the rELISA assay for anti-SLA/LP as well as a random sample of 50 negative sera were tested by confirmatory western blotting. Western blot was performed as described previously using the recombinant SLA/LP antigen. ${ }^{16}$ SLA/LP antigen $(0.6 \mu g)$ was separated on $10 \%$ SDS-PAGE using a Mini Protean II electrophoresis chamber (BioRad, Munich, Germany), and proteins were transferred to a polyvinylidene difluoride membrane (Millipore, Eschborn, Germany). Membranes were then incubated with patient sera (diluted 1:200 in 2\% milk powder/ PBST) or with a monoclonal, mouse derived anti-penta-his antibody (diluted 1:1500 in 3\% BSA/PBS; Qiagen, Hilden, Germany) that recognises histidine pentamers. After washing, membranes were incubated for one hour with a peroxidase conjugated antibody to human IgG, IgA, IgM, and kappa and lambda proteins (Dako, Copenhagen, Denmark; diluted $1: 1000$ in $2 \%$ milk powder/PBST) or anti-mouse IgG (Dako; diluted $1: 1000$ in $2 \%$ milk powder/PBST). Staining was performed using the metal enhanced diaminobenzidine kit (Boehringer, Mannheim, Germany). In addition, commercially available standardised western blot membranes with recombinant SLA/LP antigen (Euroimmun, Groß Grönau, Germany) were developed and tested. Incubation with patient sera (diluted 1:50) for one hour at room temperature, washing, incubation with alkaline phosphatase marked goat derived antihuman IgG, and staining with substrate solution were performed according to the manufacturer's instructions.

\section{Statistical analysis}

The Mann-Whitney test was used to compare non-parametric variables in independent samples. Fisher's exact test was used to compare the frequencies of dichotomous variables and the unpaired $t$ test was used to compare the significance of differences in the means of continuous variables. As the variables for comparison had been formulated a priori and then assessed systematically in each group, an unadjusted $\mathrm{p}$ value of 0.05 was used to determine statistical significance. Data are presented as mean (SEM) in the tables and text.

\section{RESULTS}

Sensitivity and specificity of SLA/LP immunoassays in patients with autoimmune hepatitis

A total of 454 serum samples taken from 419 patients who were evaluated for AIH in four different centres (Brazil, Germany, Japan, and the USA) were tested under code by the new anti-SLA/LP rELISA, the original iELISA, and by western blotting. Figure 2 shows the results of immunoblotting analysis of representative sera, and fig 3 summarises the results of the two immunoassays. Among the 454 serum samples from 419 patients with AIH, there was only one false positive test by 
Table 1 Clinical features and treatment outcomes in American, German, and Japanese patients with autoimmune hepatitis with and without antibodies to soluble liver antigen/liver pancreas (SLA/LP) antigen

\begin{tabular}{|c|c|c|c|c|c|c|c|c|c|c|}
\hline \multirow[b]{3}{*}{ AST $1 \mathrm{U} / \mathrm{mll}$} & \multicolumn{4}{|l|}{ USA } & \multicolumn{4}{|c|}{ Germany } & \multicolumn{2}{|l|}{ Japan } \\
\hline & \multicolumn{2}{|c|}{$\begin{array}{l}\text { SLA/LP positive } \\
(n=23)\end{array}$} & \multicolumn{2}{|c|}{$\begin{array}{l}\text { SLA/LP negative } \\
(n=126)\end{array}$} & \multicolumn{2}{|c|}{$\begin{array}{l}\text { SLA/LP positive } \\
(n=21)\end{array}$} & \multicolumn{2}{|c|}{$\begin{array}{l}\text { SLA/LP negative } \\
(n=87)\end{array}$} & $\begin{array}{l}\text { SLA/LP positive } \\
(n=2)\end{array}$ & $\begin{array}{l}\text { SLA/LP negative } \\
(\mathrm{n}=28)\end{array}$ \\
\hline & 442 & (78) & 507 & (37) & 186 & (47) & 275 & (27) & $51 \quad(15.5)$ & $227 \quad(62)$ \\
\hline$\gamma$ globulinst & & $(0.2) \mathrm{g} / \mathrm{dl}$ & & $(0.1) \mathrm{g} / \mathrm{dl}$ & 27 & $(1.6) \%$ & 31 & (1)\% & $34 \quad(2) \%$ & $30 \quad(1.9) \%$ \\
\hline Bilirubin (mg/dl) & 4.9 & $(1.20)$ & & $(0.5)$ & 3.2 & (1.5) & & (0.6) & $0.8(0.4)$ & $2.5(0.6)$ \\
\hline Age at diagnosis (y) & 43 & (2) & 46 & (2) & 41 & (3) & 46 & (2) & $48 \quad$ (4) & $54 \quad(3.1)$ \\
\hline $\operatorname{Sex}(M: F)$ & $5: 18$ & & $26: 100$ & & $2: 19$ & & $11: 76$ & & $0: 2$ & $2: 26$ \\
\hline Cirrhosis at diagnosis & $7 / 23$ & $(30 \%)$ & $33 / 124$ & $(27 \%)$ & $8 / 20$ & $(40 \%)$ & $27 / 71$ & $(38 \%)$ & 0 & na \\
\hline Remission & $15 / 22$ & $(68 \%)$ & $88 / 121$ & $(73 \%)$ & 21 & $(100 \%)$ & $67 / 81$ & (83\%) & na & na \\
\hline Relapse after remission & $15 / 15$ & $(100 \%)^{*}$ & $64 / 88$ & $(73 \%)^{*}$ & $11 / 20$ & $(55 \%)^{*}$ & $28 / 82$ & $(34 \%)^{*}$ & na & na \\
\hline Duration of symptoms (months) & 38 & (11) & 27 & (4) & 35 & (9) & 26 & (10) & na & na \\
\hline
\end{tabular}

*Statistical difference between SLA/LP positive (26 of 35) and SLA/LP negative (92 of 170) at $p=0.02$

†Units in US patients in $\mathrm{g} / \mathrm{dl}$, in German and Japanese patients as relative per cent (\%) of total serum protein (absolute values not available in all patients). AST, aspartate aminotransferase; na, not available.

Values are mean (SEM) or number (\%).

the rELISA based on recombinant antigen; this sample tested negative in western blotting and in the iELISA. The specimen showed non-specific binding to ELISA plates and gave false positive results in various other ELISA, including those for antimitochondrial antibodies and anti-LKMI.

Within the group of 21 sera showing borderline reactivity in the rELISA assay, nine sera tested clearly positive by western blot analysis. Four of the 21 borderline sera showed weakly positive staining by western blot and thus were considered weakly positive for anti-SLA/LP; the other eight of the 21 sera with borderline reactivity in the rELISA appeared negative by western blotting and were considered anti-SLA/LP negative. Of the 67 sera, which were positive by rELISA and western blot, the original iELISA detected only 61 ; in addition, the iELISA missed the four borderline positive sera, as defined by rELISA and western blot. The inhibition based ELISA did not have a false positive result.

Considering the 454 sera from four countries and the 1026 sera tested for validation of the new assay, the overall specifi- city of the rELISA for anti-SLA/LP was $99 \%$ if the higher cut off value was applied (upper limit of borderline reactivity 30 $\mathrm{RU} / \mathrm{ml})$. The sensitivity of the assay using the same cut off value was $87 \%$ ( 10 of 77 sera were falsely negative for SLA/LP). By using the lower cut off value $(8 \mathrm{RU} / \mathrm{ml})$, the sensitivity of the rELISA for SLA/LP increased to $100 \%$ but specificity was reduced to $83 \%$. By combining the rELISA for anti-SLA/LP with confirmatory western blotting of all borderline sera, specificity was $99 \%$ and sensitivity was $100 \%$.

\section{Frequency of anti-SLA/LP in AlH from different countries}

Antibodies to SLA/LP were detected in 23 of 149 patients who had been diagnosed with AIH at the Mayo Clinic in the USA (15\%). Antibodies to SLA/LP were detected in 23 serum specimens from 132 patients evaluated in Sao Paulo, Brazil (17\%); in two serum specimens from 30 patients evaluated in Kagawa, Japan (7\%); and in 21 serum specimens from 108 patients evaluated in Mainz, Germany (19\%). Among the 69

\begin{tabular}{|c|c|c|c|}
\hline Clinical features & $\begin{array}{l}\text { AlH type } 1 \text { SLA/LP } \\
\text { positive }(n=23)\end{array}$ & $\begin{array}{l}\text { AlH type } 1 \text { SLA/LP } \\
\text { negative }(n=93)\end{array}$ & $\begin{array}{l}\text { AlH type } 2 \\
(n=16)\end{array}$ \\
\hline \multicolumn{4}{|l|}{ Skin colour } \\
\hline White & $12(52 \%)$ & $37(40 \%)$ & $10(63 \%)$ \\
\hline Mulatto & $9(39 \%)$ & $38(41 \%)$ & 5 (31\%) \\
\hline Black & $2(8 \%)$ & $14(15 \%)$ & $1(6 \%)$ \\
\hline Japanese descendant & 0 & $4(4 \%)$ & \\
\hline \multicolumn{4}{|l|}{ Sex } \\
\hline Male & 0 & $19(20 \%)$ & $2(13 \%)$ \\
\hline Female & 23 & $74(80 \%)$ & $14(88 \%)$ \\
\hline \multicolumn{4}{|l|}{ Age of onset (y) } \\
\hline $0-15$ & $7(30 \%)$ & $44(47 \%)$ & 11 (69\%) \\
\hline $15-30$ & $5(22 \%)$ & $17(18 \%)$ & $4(25 \%)$ \\
\hline $30-50$ & $11(48 \%)$ & $19(21 \%)$ & $1(6 \%)$ \\
\hline $50-70$ & 0 & $13(14 \%)$ & 0 \\
\hline \multicolumn{4}{|l|}{ AST $(\times N V)$} \\
\hline $0-5$ & $5(22 \%)$ & $11(12 \%)$ & $3(19 \%)$ \\
\hline $5-10$ & $4(17 \%)$ & $11(12 \%)$ & $3(19 \%)$ \\
\hline$>10$ & $14(61 \%)$ & $71(76 \%)$ & $10(62 \%)$ \\
\hline \multicolumn{4}{|l|}{$\gamma$-globulins (xNV) } \\
\hline $1.0-1.5$ & $2(9 \%)$ & 15 (16\%) & $3(19 \%)$ \\
\hline $1.5-2.0$ & $6(26 \%)$ & $17(18 \%)$ & $8(50 \%)$ \\
\hline$\geqslant 2.0$ & $15(65 \%)$ & $61(66 \%)$ & $5(32 \%)$ \\
\hline \multicolumn{4}{|l|}{ Total bilirubins (mg\%) } \\
\hline $0-5$ & $17(74 \%)$ & $38(41 \%)$ & $6(38 \%)$ \\
\hline $5-10$ & $5(22 \%)$ & $29(31 \%)$ & $5(31 \%)$ \\
\hline$>10$ & $1(4 \%)$ & $23(25 \%)$ & $5(31 \%)$ \\
\hline Cirrhosis at diagnosis & $8 / 17(47 \%)$ & $68 / 93(63 \%)$ & $8 / 14(57 \%)$ \\
\hline
\end{tabular}


specimens positive for anti-SLA/LP, 18 (26\%) had been negative for ANA and SMA, including two from the Mayo Clinic, six from Brazil, one from Japan, and nine from Germany. The frequency of seropositivity for anti-SLA/LP was determined by rELISA and western blotting.

\section{Clinical characteristics associated with anti-SLA/LP}

Patients whose sera were positive for anti-SLA/LP by the rELISA had clinical features that were similar to those whose sera were negative for anti-SLA/LP (tables 1, 2). Furthermore, there were no distinctions based on country of origin. Age at diagnosis and sex distribution were similar, as were serum levels of bilirubin and $\gamma$-globulin in patients with and without anti-SLA/LP. Serum aminotransferase levels tended to be lower in seropositive patients in all four countries but these differences were not statistically significant. Likewise, the slightly higher frequency of cirrhosis at presentation in patients with anti-SLA/LP did not distinguish them. Additionally, there were no differences between the 18 patients with anti-SLA/LP who tested negative for ANA and SMA and the 53 patients with anti-SLA/LP who were also positive for ANA and/or SMA.

Autoantibodies to SLA/LP were not detected in the sera of patients with anti-LKMl. Only Brazilian patients with anti-LKMl were included in this study and they were indistinguishable from Brazilian patients with anti-SLA/LP except for their younger age (table 2).

\section{Treatment outcomes associated with anti-SLA/LP}

Relapse after induction of remission occurred in each of the 15 SLA/LP seropositive patients from the USA who entered remission and were withdrawn from corticosteroids (table 2). In contrast, significantly less seronegative patients relapsed after withdrawal of treatment $(73 \% \vee 100 \% ; \mathrm{p}=0.02)$. Similarly, the frequency of relapse after drug withdrawal was higher in seropositive patients from Germany but this difference was not statistically significant (55\% v 36\%; $\mathrm{p}=0.09$ ). Combining the American and German patients, the relapse rate for SLA/LP positive patients was 26/35 (74\%) compared with 92/170 (54\%) in SLA/LP negative AIH $(p=0.02)$.

\section{DISCUSSION}

Antibodies to SLA and LP, initially described independently, were found to be highly specific markers for autoimmune liver disease. ${ }^{12}{ }^{13}$ Identification of the target antigen has recently confirmed previous suggestions that these antibodies are identical. ${ }^{1622}$ We now describe for the first time an assay for these antibodies that is based on the recently identified and cloned target antigen and we demonstrate the validity and value of this immunoassay in assessing patients with AIH.

The rELISA that we developed based on the recombinant antigen showed excellent specificity and sensitivity when combined with immunoblot for confirmation of borderline positive sera. False positive results above the higher cut off value of $30 \mathrm{RU} / \mathrm{ml}$ occurred only in a single serum sample which probably contained aggregates that caused non-specific binding to ELISA plates, a well described liability of this technique. ${ }^{23}$ Furthermore, within the borderline range of positivity $(8-30 \mathrm{RU} / \mathrm{ml})$ nearly half of the serum samples lacked anti-SLA/LP by western blot. This finding indicates the importance of confirmatory testing by western blot of all borderline positive samples.

Maximum sensitivity and specificity were obtained using a range of 8-30 RU/ml to define borderline positive sera and by assessing all such sera by immunoblotting. Unfortunately, we cannot exclude the fact that some sera used in our study had been thawed previously and we can only speculate that use of fresh serum in routine medical practice may diminish the number of borderline positive results. Future prospective analyses are necessary to confirm the advantages of fresh serum specimens in the assay system and to fully evaluate the frequency and importance of double testing of all borderline positive samples.

Volkmann et al have recently described reactivity of anti-SLA/LP positive sera with a shorter version of the recombinant molecule containing the main immunogenic region at the C terminus. ${ }^{18}$ Using an earlier version of the commercially available rELISA described here, they confirmed the specificity of this assay. However, they found some false negative cases. In addition, they reported 18 sera positive by iELISA but not reactive with the recombinant protein. There may be several reasons for this apparent discrepancy. Firstly, as the iELISA uses a positive patient serum as a gold standard, and as this serum may contain other autoantibodies, the specificity of the iELISA crucially depends on the individual serum selected for the assay. Secondly, the authors apparently did not use a borderline range in the rELISA. Thirdly, additional conformational epitopes may be present on the SLA/LP protein, as suggested in a preliminary report. ${ }^{24}$ However, our own data have thus far argued against a major reactivity to conformational epitopes. ${ }^{16}$

Importantly, none of the 1026 samples obtained prospectively from patients with various liver diseases and general medical problems gave a false positive result above the borderline range. This finding attests to the specificity of the assay and anti-SLA/LP for autoimmune liver disease. In our experience to date, anti-SLA/LP have only been detected in patients with AIH or the overlap syndrome of $\mathrm{PBC}$ and AIH. ${ }^{1621}$ This was also found by others. ${ }^{12}{ }^{1525}$ Antibodies to SLA/LP were found in patients with AIH from all countries that were studied and there were no statistically significant differences in the frequencies of occurrence based on geographical region. The lowest prevalence was among patients from Japan, but this study group was small. The highest prevalence was among patients from Germany, but this finding may have reflected selection. The Mainz group has had a longstanding investigative interest in testing for anti-SLA and the availability of the iELISA for at least 10 years may have enriched the patient base with seropositive patients. ${ }^{12}{ }^{14}$ Conversely, patients with AIH and anti-SLA/LP may have been underdiagnosed in other centres, especially if they lacked ANA or SMA. This hypothesis is supported by the observation that while nine of 21 SLA/LP positive patients from Germany lacked other autoantibodies, only two of 23 patients from the Mayo clinic were similarly seronegative. Widespread testing for SLA/LP autoantibodies is likely to detect more patients with AIH who currently lack conventional autoantibodies. ${ }^{17} 2627$

The ability of anti-SLA/LP to define a clinically distinct subgroup of AIH has been controversial. ${ }^{12-14} 28-32$ Our findings indicated that patients with anti-SLA/LP did not have a distinctive clinical syndrome regardless of country of origin. Differences in serum aminotransferase levels and frequency of cirrhosis at presentation were not compelling justifications for separate designation of these patients as type 3 AIH. Seropositivity however was associated with a higher frequency of relapse after corticosteroid withdrawal in patients from the USA and Germany and the presence of anti-SLA/LP may be a prognostic index. Future studies must evaluate the prognostic value of testing for anti-SLA/LP in well characterised patients who have disease of similar severity and comparable treatment.

In summary, anti-SLA/LP can be detected reliably by a standardised rELISA based on the recombinant target antigen. Borderline positive results require further clarification by western blot. Seropositivity occurs with similar frequencies in AIH from different geographical regions and races. Antibodies to SLA/LP are present in all age groups, including young children, and they do not seem to denote a clinically distinct subgroup. However, SLA/LP autoantibodies are useful tools for the diagnosis of AIH, and it remains to be seen whether seropositivity may characterise patients who are 
more likely to relapse after corticosteroid withdrawal. Antibodies to SLA/LP are frequently the sole immunoserological markers of AIH. The assay may therefore help to identify additional patients with AIH previously thought to suffer from cryptogenic hepatitis. SLA/LP was exclusively detected in AIH making it a diagnostic test of direct clinical relevance. Availability of standardised SLA/LP immunoassays will facilitate diagnosis of AIH.

\section{ACKNOWLEDGEMENTS}

This study was supported by the Stiftung Rheinland-Pfalz für Innovation.

\section{Authors' affiliations}

M Baeres, J Herkel, I Wies, S Kanzler, P R Galle, A W Lohse,

I Department of Medicine, Johannes Gutenberg-University, Mainz,

Germany

A J Czaja, Division of Gastroenterology and Hepatology, Mayo Clinic, Rochester, Minnesota, USA

E L R Cancado, G Porta, Division of Gastroenterology, University of Sao Paulo, Sao Paulo, Brazil

M Nishioka, Department of Medicine, Kagawa Medical School,

Kagawa, Japan

T Simon, Diarect AG, Freiburg, Germany

C Daehnrich, W Schlumberger, Euroimmun GmbH, Groß Grönau, Germany

\section{REFERENCES}

1 Gordon SC. Diagnostic criteria, clinical manifestations and natural history of autoimmune hepatitis. In: Krawitt EL, Wiesner RH, Nishioka M eds. Autoimmune liver diseases. Amsterdam: Elsevier, 1998:343-60.

2 Alvarez F, Berg PA, Bianchi FB, et al. International Autoimmune Hepatitis Group report: review of criteria for diagnosis of autoimmune hepatitis. J Hepatol 1999;31:929-38.

3 Lohse AW, Gerken G, Mohr H, et al. Distinction between autoimmune liver diseases and viral hepatitis: clinical and serological characteristics in 859 patients. Z Gastroenterol 1995;33:527-33.

4 Homberg JC, Abuaf N, Bernard O, et al. Chronic active hepatitis associated with anti liver/kidney microsome antibody type 1: a second type of "autoimmune" hepatitis. Hepatology 1987;7:1333-9.

5 Lunel F, Abuaf N, Frangeul L, et al. Liver/kidney microsome antibody type 1 and hepatitis $C$ virus infection. Hepatology 1992;16:630-6.

6 Yamamoto AM, Cresteil D, Homberg JC, et al. Characterization of the anti-liver-kidney microsome antibody (anti-LKM1) from hepatitis C virus-positive and -negative sera. Gastroenterology 1993;104:1762-7.

7 Gerotto M, Pontisso P, Giostra F, et al. Analysis of the hepatitis C virus genome in patients with anti-LKM-1 autoantibodies. J Hepatol 1994:21:273-6.

8 Mackie FD, Peakman M, Yun M, et al. Primary and secondary liver/kidney microsomal autoantibody response following infection with hepatitis C virus. Gastroenterology 1994;106:1672-5.

9 Durazzo M, Philipp T, van Pelt FNAM, et al. Heterogeneity of microsomal autoantibodies (LKM) in chronic hepatitis $C$ and $D$ virus infection. Gastroenterology 1995;108:455-62.

10 Giostra F, Manzin A, Lenzi M, et al. Low hepatitis $C$ viremia in patients with anti-liver/kidney microsomal antibody type 1 positive chronic hepatitis. J Hepatol 1996;25:433-8.

11 Lohse AW, Obermayer-Straub P, Gerken G, et al. Development of cytochrome P450 2D6-specific LKM-autoantibodies following liver transplantation for Wilson's disease. Possible association with a steroid-resistant transplant rejection episode. J Hepatol 1999;31:149-55.

12 Manns M, Gerken G, Kyriatsoulis A, et al. Characterization of a new subgroup of autoimmune chronic active hepatitis by autoantibodies against a soluble liver antigen. Lancet 1987;1:292-4.

13 Stechemesser E, Klein R, Berg PA. Characterization and clinical relevance of liver-pancreas antibodies in autoimmune hepatitis. Hepatology 1993;18:1-9.

14 Kanzler S, Weidemann C, Gerken G, et al. Clinical significance of antibodies to soluble liver antigen in autoimmune hepatitis. J Hepatol 1999:31:635-40.

15 Ballot E, Homberg JC, Johanet C. Antibodies to soluble liver antigen: an additional marker in type 1 auto-immune hepatitis. J Hepatol 2000;33:208-15

16 Wies I, Brunner S, Henninger J, et al. Identification of target antigen for SLA/LP autoantibodies in autoimmune hepatitis. Lancet 2000;355:1510-15.

17 Czaja AJ, Homburger HA. Antibodies in liver disease. Gastroenterology $2001 ; 120: 239-49$

18 Volkmann M, Martin L, Baurle A, et al. Soluble liver antigen: isolation of a 35-kd recombinant protein (SLA-p35) specifically recognizing sera from patients with autoimmune hepatitis. Hepatology 2001;33:591-6.

19 Gelpi C, Sontheimer EJ, Rodriguez-Sanchez JL. Autoantibodies against a serine tRNA-protein complex implicated in cotranslational selenocysteine insertion. Proc Natl Acad Sci USA 1992;89:9739-43

20 Costa M, Rodriguez-Sanchez JL, Czaja AV, et al. Isolation and characterization of cDNA encoding the antigenic protein of the human tRNP(Ser)Sec complex recognized by autoantibodies from patients with type-1 autoimmune hepatitis. Clin Exp Immunol 2000;121:364-74.

21 Lohse AW, Meyer zum Büschenfelde KH, Kanzler FB, et al. Characterization of the overlap syndrome of primary biliary cirrhosis (PBC) and autoimmune hepatitis: evidence for it being a hepatitic form of PBC in genetically susceptible individuals. Hepatology 1999:29:1078-84.

22 Klein R, Berg PA. Glutathione S-transferase as a major autoantigen in anti-SLA-positive autoimmune hepatitis. Gastroenterology 1999:116:1015-16.

23 McFarlane IG, Smith HM, Johnson PJ, et al. Hepatitis $C$ virus antibodies in chronic active hepatitis: pathogenetic factor or false-positive result? Lancet 1990;335:754-7.

24 Ma Y, Bogdanos DP, Williams R, et al. Anti-SLA antibodies preferentially recognise conformational epitopes in autoimmune liver disease. J Hepatol 2001;34(suppl 1):210.

25 Ma Y, Okamoto $M$, Thomas $G$, et al. Autoantibodies to soluble liver antigen/UGA suppressor tRNA-associated antigenic protein are specific to autoimmune liver disease. Hepatology 2000;32(suppl):310A.

26 Czaja AJ, Carpenter HA, Santrach PJ, et al. The nature and prognosis of severe cryptogenic chronic active hepatitis. Gastroenterology 1993:104:1755-61.

27 Kaymakoglu S, Cakaloglu Y, Demir K, et al. Is severe cryptogenic chronic hepatitis similar to autoimmune hepatitis? J Hepatol 1998;28:78-83.

28 Stechemesser E, Strienz J, Berg PA. Serological definition of a new subgroup of patients with autoimmune chronic active hepatitis. Lancet 1987;1:683.

29 Czaja AJ, Carpenter HA, Manns MP. Antibodies to soluble liver antigen, P450IID6, and mitochondrial complexes in chronic hepatitis. Gastroenterology 1993;105:1522-8.

30 Czaja AJ, Manns MP. The validity and importance of subtypes of autoimmune hepatitis: a point of view. Am J Gastroenterol 1995;90:1206-11.

31 McFarlane IG. The relationship between autoimmune markers and different clinical syndrome in autoimmune hepatitis. Gut 1998:42:599-602.

32 McFarlane IG. Lessons about antibodies in autoimmune hepatitis. Lancet 2000;355:1475-6. 